\title{
AN INTEGRATIVE MODEL AND DYNAMIC NOSOLOGY OF PERSONALITY DISORDER: PART 2: SYMPTOM-BASED PHARMACOTHERAPY
}

\author{
Dragan Svrakic $^{1}$, Mirjana Divac-Jovanovic ${ }^{2} \&$ Naazia Azhar ${ }^{1}$ \\ ${ }^{I}$ Department of Psychiatry, VA medical center St Louis, Washington University School of Medicine, St Louis, MO, USA \\ ${ }^{2}$ Department of Psychology, Faculty for Media and Communication, Singidunum University, Belgrade, Serbia
}

received: 28.12.2018;

revised: 15.2.2019;

accepted: 26.2.2019

\section{SUMMARY}

This paper presents an integrative model of personality and personality disorder which incorporates psychoanalytic concepts with modern neuroscience. In addition, a dynamic, personalized, and context - and time-sensitive diagnosis of personality disorder is introduced. The authors cogently argue that all clinical variants of personality disorder share the same common deficit: fragmented basic units of experience at the nonconscious core of the mind (aka "partial object relations"). The fragmentation propagates through mental faculties (thought, motivation, emotion), as they self-organize into subsystems of personality, e.g., one's sense of self, identity, character, moral values, rendering them polarized into extreme and thus adaptively suboptimal. The syndrome of personality disorder arises as a nonconscious compensatory maneuver of the fragmented mind to organize itself through a defensive but unrealistic self-image (e.g., narcissistic, schizoid, antisocial, etc.), giving rise to a host of unique symptoms. Symptomatic pharmacotherapy of personality disorder is best organized around four empirically derived domains of symptoms, shared by all variants to a variable degree: i) mood and anxiety dysregulation; ii) impulsivity, aggression, and behavior dyscontrol; iii) emotional disinterest and detachment; and iv) cognitive distortions and brief reactive psychoses. Pharmacotherapy targeting the above domains is nonspecific, as medications affect multiple domains simultaneously. Modest empirical evidence and considerable clinical benefits continue to support the use of medications in the overall symptomatic treatment of personality disorder.

Key words: personality disorder - pharmacotherapy - integrative model - temperament - character

$* * * * *$

\section{INTRODUCTION}

The most important goal of this paper is to challenge the therapeutic nihilism which surrounds the syndrome of personality disorder. These individuals are widely considered "untreatable", even by many mental health professionals. We argue that they can be effectively treated, but the success requires expertise and time: a precise $^{1}$ diagnosis (not only of clinical symptoms but also of the underlying organization of the mind $)^{2}$, individualized treatment planning (combining expert pharmacotherapy and in-depth psychotherapy), a patient determined to change (preferably one who trusts psychological mechanisms to achieve change), and an expert clinician with a genuine interest in this type of psychopathology, who is skilled in both psychotherapy and pharmacotherapy or, alternatively, a close collaboration between an expert psychotherapist and a skilled psychopharmacologist.

\footnotetext{
1 Irresponsible diagnosing of every loud, belligerent, or manipulative individual as personality disorder has in part created the untreatability myth of the bona fide cases

${ }^{2}$ Mind, mental (mens = Latin for mind), and personality are equivalent concepts: personality is the unique configuration of the mind in a given individual, as discussed in the text
}

\section{AN INTEGRATED, PERSONALIZED, AND DYNAMIC CONCEPT OF PERSONALITY DISORDER}

The human mind is best conceptualized as a progressively differentiated brain's adaptive agency that mediates the brain's dynamic and interactive communication with internal and external environments (Proust 2009). This fundamental adaptive function of the mind is subserved by mental faculties of thought, emotion, and motivation, also called "mental trilogy". As a selforganizing complex adaptive system, the mind develops through punctuated homeostasis, with periods of relative stability (maintained by homeostasis) followed by rather abrupt transitions (homeostatic optimization) towards increasingly complex organization and functioning (called development) (Svrakic et al. 1996). These abrupt transitions occur whenever the mind, configured to function optimally in a given set of conditions, becomes suboptimal to meet increasingly complex or different adaptive tasks. For illustration, temperament, which is the main and perfectly adequate avenue to communicate needs to the environment during the first year of life, becomes adaptively suboptimal in the context of subsequent more complex interactions. At this point, an adaptively fitter subsystem, the sense of self, abruptly self-organizes within the mind at around 2-3 years of life. 


\section{The neuroscience of psychoanalysis: a primer}

Under normal circumstances, the early mind is polarized because negative (frustrating) and positive (pleasurable) experiences are engramized by anatomically separate subcortical regions. However, the maturing general network of cognition ${ }^{3}$ eventually becomes capable to functionally ${ }^{4}$ bridge the separated extremes into a realistic, balanced understanding of self and others as sometimes "bad" but predominantly "good" ("whole object relations"). We refer to object relations as "elementary particles of the mind", because each encompasses a memorized perception of an object, the related self-reflection, and the connecting affect. This functional union is not divisible to lower order units of mental experience, only to first order sensory percepts and subcortical affects. Others have referred to object relations as the "basic units of all experience" formed from fantasy and real early interactions (Auchincloss \& Samberg 2012).

Based on LeDoux and Brown (2017) we postulate that object relations, whether partial or whole, operate within nonconscious working memory. To elaborate, all sensory events, factual- and autobiographical memories, and their respective affects are first order represented in secondary sensory cortical areas, or engramized in the medial temporal lobe and the amygdala, respectively. From here, they are "pulled" into nonconscious working memory by the general network of cognition as higher order-representations (HOR) but are still nonconscious (nonconscious HOR of first order processing) (LeDoux $\&$ Brown 2107). From nonconscious working memory, these basic units inform ${ }^{5}$ the development of mental faculties (thought, emotion, and motivation), and thus fundamentally influence their nature (balanced in normal development, polarized into extremes in personality disorder). The described sequence whereby sensory events and affects are first higher order represented into nonconscious working memory is a necessary step before cognitive networks can process them into being conscious. Thus, the nonconscious working memory harboring nonconscious affective-cognitive elementary

\footnotetext{
${ }^{3}$ General network of cognition (GNC) includes circuits spread across frontal and parietal areas: anterior cingulate cortex, lateral and medial orbital frontal cortex, dorsolateral, dorsomedial, ventrolateral, and ventromedial prefrontal cortex (PFCx). GNC is implicated in the development of cognitive processes of attention, working memory, and metacognition, which are important in the development of conscious experiences (for details see LeDoux \& Brown 2017).

${ }^{4}$ The merging into whole object relations is not structural, but a function of working memory, most likely nonconscious working memory.

${ }^{5}$ Serve as the rudiments upon which more complex thought, emotion, and motivation are built. They are integrated into these mental faculties and thus and thus cannot be conscious themselves, only indirectly recognized by the effects of their operations (e.g., mature vs extremized interpersonal reltions or self-image)
}

units of experience can be thought of as the "core" of the mind. How exactly the subsystems of the mind come to existence is not known. A straightforward speculation is that during the progressive differentiation of the mind, reflecting self-organizing multidirectional optimization processes to meet the increasing adaptive demands before it, the brain's functional networks involved in thought, emotion, and motivation self-organize into semi-stable configurations which we refer to as the subsystems of the mind: contextualized temperament, the sense of self, character, moral standards (both advancing social adaptation), and identity (the assuring "who" of "me" which provides the sense of consistency and thus enables planning for the future). Each of these subsystems emerges canonically at a specific developmental phase and each provides a specific adaptive function to the system of the mind as a whole.

Observation of infants and children provides the timeline for personality (mind) development. The nonconscious self-HORs, based on body sensations and rudimentary self-reflections within nonconscious working memory, distinguish self ("I") from other ("not I") around 6 months of age. It is a mental state "but in an extremely thin sense" (LeDoux \& Brown 2017) insofar as it does not invoke self- awareness. From here, the nonconscious self-HORs are re-represented by cognitive networks (HOR of HOR or HOROR) which makes them accessible by passive noticing as "phenomenally conscious" sense of self ("me" and "mine") around 2-3 years of age. Phenomenal consciousness is best described as "knowing how it feels", or as "what is it like" to have a first person experience (the taste of strawberries, the feeling of safety with the mother around, etc.) (LeDoux \& Brown 2017). Such passive experience is not accessible by active scrutinizing (introspection).

For active introspection, another cognitive re-representation (HOR of HOROR) is needed, for which cognitive networks become capable around 3-5 years of age, paving the way for the emergence of the sense of self as intentional and purposeful ("me and my intentions") which is actively accessible by introspection (aka "selfawareness" or "autonoetic consciousness") ${ }^{6}$. Around the same time (cca. 5 years of age), maturing cognitive networks enable the emergence of theory of mind of others, capacity for mentalization (McAdams 2017) and capacity for autobiographical memory. The latter underlies the sense of identity, which allows the experience of continuity in time and space ("me, my past-, present-, and- future") (reviewed in detail in Svrakic \& DivacJovanovic 2018). The exact mechanism of cognitive representations and re-representations is not known. What is known is that they are functional (not structural) (emerging functions by the work of GNC), but in all likelihood involve some kind of rapid and neuroplasticity. Thus the subsystems of the mind are semistable.

\footnotetext{
${ }^{6}$ LeDoux \& Brown (2017) provide an excellent discussion on the subject.
} 
The postulated existence of nonconscious working memory by cognitive neuroscience opens the door to reconcile psychoanalytic observations by M. Klein (the founder of object relations theory) with modern neuroscience. As discussed, operating within nonconscious working memory, internalized object relations fundamentally inform mental development. Because these core early experiences are highly personal, personality arises as a unique configuration of the mind in a given individual, a concept supported by functional neuroimaging (Gratton et al. 2018). Incidentally, defense mechanisms, we believe, operate within the noncomscious working memory as a homeostatic function of the mind.

\section{Developmental homeostasis of personality disorder}

Personality disorder follows the same developmental path as normal personality would, but here in the context of heritably excessive temperament traits ("trait vulnerabilities") and/or adverse environments ("bad-enough environments"). These overwhelming risk factors, singly or in concert, interfere with the maturation of prefrontal functional networks and disrupt normal mental development, both well documented aberrances in personality disorder. As a result, polarized "either-or" elementary units of the mind, aka "partial object relations" pathologically persist at the nonconscious core of the mind. The persisting polarization protects the few positive experiences which are in minority, and thus endangered to be annihilated, but are vital for mind development. It is unclear whether the continuing splitting reflects a nonconscious defense mechanism (psychoanalytic interpretation) or stress-related hypofunctional PFCx (neuroscience interpretation $)^{7}$, or perhaps the former is simply a function of the latter. However, as a phylogenetic given, a mind and its subsystems have to develop in a human being, and this even with defective building components. This is known as "developmental homeostasis", a biological process in which all animals develop more or less normally within a range, despite some polymorphic genes and less than perfect environments. In personality disorder, mental development is informed by fragmented elementary particles and this renders personality adaptively suboptimal. We have empirically shown that borderline ${ }^{8}$ (i.e., fragmented) personality

\footnotetext{
${ }^{7}$ Stress affects PFCx network development and topology in two ways: mild stress drives random brain networks toward scale- free, crash- resistant connectivity, whereas severe and persistent stress may interfere with the development of scalefree connectivity, even fracture the already established ones (Zorumski \& Rubin 2011). Sych hypofrontality has been documented in personality disorder.

${ }^{8}$ We advocate that the term "borderline" be replaced with the term "fragmented" personality, as the latter highlights intrapsychic fragmentation as the essence of personality disorder. The term "borderline" has been overused and misused to the point of confusion, as a type of personality organization (Kernberg), to denote any "difficult" patient
}

represents a shared impairment, a common denominator cutting across all clinical variants of personality disorder (Divac-Jovanovic et al. 1993). The syndrome arises as a homeostatic maneuver of the early mind to compensate for its fragmentation through uncontested (omnipotent, wishful) defensive fantasy, the latter, by brute force, unifies the fragments into a fantasized defensive ideal. This nonconscious strategy gives rise to a highly unrealistic yet a more stable self-image that evades disintegration but further pathologizes personality by funnelling it into deviance. The latter varies with clinical variants of personality disorder, each with unique maladaptive behaviors which express the corresponding deviant self-image, e.g., antisocial, schizoid, narcissistic, histrionic, paranoid, negativistic dependent, and anankastic, although mixed presentations are the most frequent. Such an adaptively suboptimal personality continues to vitally need sources of organization, both internally (through fantasy and exaggeration) and interpersonally (through a continual need for selfobjects). The latter are expected, frequently demanded, to define and maintain the unrealistic self-image of the patient, hence the frequent interpersonal conflicts.

Personality disorder is not a static, lifelong diagnosis as is commonly believed. The average short-term testretest reliabilities are 0.54 for specific subtypes and 0.56 for any personality disorder, even with structured interviewing (McGlashan et al. 2005). Longer term testretest reliabilities are found to be 0.51 for any personality disorder and 0.34 for specific subtypes, which, together with significant diagnostic change over as little as six months, indicate a diagnostic reversibility of the syndrome (reviewed in APA 2012). However, personality disorder is not quite a state condition as well, but is captured by the concept of semi-stability: it fluctuates with changing internal or external conditions, both stabilizing and destabilizing. Incidentally, the same is true of normal personality. Such semi-stability calls for a dynamic nosology of personality and personality disorder. In contrast to current static classifications (DSM, ICD) and other popular concepts of personality pathology (Kernberg, Millon) we have developed a dynamic and personalized diagnostic approach within a 3D diagnostic space in which each individual is uniquely positioned: two intersecting dimensions, one vertical, representing the person's qualitative level of level of mind integration or, as we call it, "level of mental functioning" (the "how" of personality), and one horizontal, representing his or her maladaptive behavior style (the "what" of personality) are cross matched in the given time and context. Such dynamic nosology is inherently sensitive to fluctuations in mental functioning over time and context, and gives the clinician precise milestones for monitoring progress in therapy.

(most clinicians), or to a separate category of personality disorder (DSM), the last two concepts plagued by stigma and therapeutic nihilism. 


\section{TREATMENT OF PERSONALITY DISORDER: TOP-DOWN AND BOTTOM-UP}

Both brain and mind aberrances characterize personality disorder: the former refer to hypofunctional cognitive networks and hyper-reactive subcortical affects, the latter to persisting primitive defense mechanisms and polarized object relations (reviewed in Svrakic \& Divac-Jovanovic 2018, Kernberg 2015). In such pathological triad of hypofrontality, hyper subcorticality, and polarized mind, the components perpetuate each other in a vicious cycle. As both cortical and subcortical processes contribute to personality disorder, the treatment can approach the problem from two directions: i) topdown approach, which aims to improve cognitive control of heightened subcortical reactivity, or ii) bottom-up approach, which aims to balance the impact of subcortical affects on the mind as a whole. The two approaches inevitably engage in a positive feedback whereby improved subcortical affects facilitate self-organization of more mature prefrontal networks and more effective cognitive (executive) functioning and vice versa. At this point, the healing of the brain/mind is not limited to treatment sessions but continues through interactive experiences in non-supervised real-life situations, e.g., within a relationship with an unconditionally loving mature partner, a rare but fortunate milieu for the fragmented partner.

Improvement of the cognitive top-down control, presumably reflecting maturation of prefrontal networks, has been thus far the domain of psychotherapy. As discussed in part I of this paper, the most effective psychotherapy is psychodynamic or psychodynamically oriented, as these provide the best fit between the healing ingredient of therapy (i.e., corrective emotional experience) and the postulated core impairment of personality disorder (i.e., polarized object relations) (review in Svrakic \& Divac-Jovanovic 2018). The following point is noteworthy: we understand the patient through transference and countertransference but heal him or her through the therapeutic relationship, as the latter provides corrective emotional experience, akin to "reparenting", which recreates the natural milieu for mind development. More recently, there has been a renewed interest in pharmacological modulation of cortical functions, using agents classified as psychedelics ${ }^{9}$, like empathogen-entactogens serotonin releasers (LSD, psilocybin) or dissociatives related to glutamatergic mechanisms (like ketamine). Several reputable researchers are reporting that psychedelics, administered to an

\footnotetext{
9 The term means "soul manifesting" in English, as these compounds cause an altered state of consciousness and sensory changes, produce experiences of emotional communion, oneness, relatedness, and emotional openness (empathogens or entactogens) and are believed to promote development of the unused potentials of the human mind (entheogens).
}

individual prepared in psychotherapy, may induce profound and lasting new insights, increased empathy, and advanced sense of well-being. These positive changes are especially strong in subjects who reported an initial experience of ego dissolution, i.e., that their sense of being a self or 'I' distinct from the rest of the world has diminished or altogether dissolved, as well as in the subjects who were listening to music during the LSD administration (hence, some have called the process as "finding the self by losing the self"). The changes are believed to be related to a resetting of functional cortical networks, much as the effects of in-depth psychotherapy. Routine clinical use of psychedelics needs further systematic testing. What appears certain at this point is that candidates for psychedelic pharmacotherapy have to be prepared with multiple sessions of preparatory psychotherapy.

The bottom-up approach which aims to correct the over-influence subcortical affects on cognition and behavior historically has been the domain of pharmacotherapy. Such heightened affect reactivity can be treated within the theoretical context of heritably excessive temperament traits, which not only act as risk factors or "trait vulnerabilities" for personality disorder but also continue to perpetuate the core psychopathology of fragmentation. Thus, pharmacotherapy of excessive temperament is bona fide "mechanism-based" (Svrakic et al. in prep.). Pharmacotherapy can also aim to correct clinical symptoms of personality disorder arranged into domains by co-occurrence. Here, we focus on the symptom-domain based pharmacotherapy, which is the standard of care at the moment. Both mechanism-based and symptom-based improvement in disruptive affects and behaviors are expected to facilitate one's capacity for engage in psychotherapy, although via fundamentally different impacts, as discussed later. What is explicit in our concept of personality disorder is that pharmacotherapy, whether mechanism-based or symptom-domain based, is unlikely to be effective unless combined with psychotherapy, i.e., that a combined topdown and bottom-up approaches provide maximal effectiveness. Of note, mechanism-based and symptombased pharmacotherapies occasionally overlap (Svrakic et al. in prep.).

\section{SYMPTOMS OF PERSONALITY DISORDER REFLECT WIDESPREAD NEURAL AND MENTAL DEFICIENCIES}

Phenomenology and symptoms of personality disorder arise from the nonconscious, "core" fragmentation of the mind: cognition, conscious emotion, motivation as well as personality susbsystems are self-organized from and operate with fragmented (unbalanced) elementary particles, as discussed. In the context of inner polarization, negative or positive stimulations (failures or approvals) easily activate negative and positive affects (anger or idealization) and the corresponding contradictory images of one's own self and others 
("bad" or "good"). Such heightened reactivity gives rise to the so called "stable instability" of personality disorder, which is observable in mental faculties (thought, motivation, emotion), as well in higher order subsystems, e.g., unstable sense of self, fluctuating identity, turbulent interpersonal relations which oscillate between devaluetion and idealization frequently of the same person, among others. In fact, the only relatively stable subsystem of the mind is excessive temperament, which in most cases has contributed to the syndrome in the first place and continues to perpetuate it.

As discussed, personality disorder arises from the homeostatic attempt of the early mind to compensate for the fragmentation and organize through defensive omnipotent fantasies. Consequently, the mind is self-organized into an unrealistic and pathological yet a more stable self-image, basically an impersonation of the fantasized defensive image (e.g., narcissistic, histrionic, antisocial, etc. The process accounts for the duality of the self-subsystem in personality disorder. The dominant compensatory self brings about behavior styles traditionally recognized as clinical variants of personality disorder: narcissistic grandiosity, bullying antisociality, histrionic theatricality, passive-aggressive dependency, paranoid distrust, schizoid detachment, and anankastic perfectionism. The dominant self-image coexists with the weaker and split-off real self (the real endangered fragmenteed mind), the latter harboring fragile self-esteem, exceptional sensitivity to rejection, feelings of inferiority, emptiness and boredom, anger and aggression, lack of genuine interest, dysphoria, paranoid fears, among other features. Figuratively, personality disorder can be thought of as "better any organization, than no organization" where an extremized unrealistic self is the lesser of two evils (the alternative is disintegration). The two selves co-exist but are barely aware of each other, divided by "vertical splitting" and mutual disavowal (Kohut 1971). Most patients are aware, albeit phenomenally only, of their own fragility and inauthenticity, which stirs up disturbing disintegration and existential anxieties as well as hypochondriasis.

Other subsystems of the mind are also affected: temperament traits are poorly contextualized and cognized ("untamed") and frequently lead the mind, character traits subserve the internal need for organization at the expense of social adaptation, identity is diffuse and changing, and moral values are opportunistic. The persisting hypofrontality may explain poor capacity of mentalization, underdeveloped theory of mind of others, impulsivity, and lack of empathy, as discussed. Kernberg (2015) describes these individuals as "reflexive" rather than "reflective".

Diagnosis of personality disorder must include both the shared aspects of fragmentation (the "how" of personality or "vertical" diagnosis, as discussed) and the overt signs and symptoms of variant-specific behavior styles (the "what" of personality or "horizontal" diagnosis), as discussed.
Stable instability of the mind: $\operatorname{mood} / \operatorname{emotions}^{10}$, thought, motivation, and behavior. Mood instability and turbulent emotions

Unpredictable swings between opposite moods, fueling matching shifts in self-image, thought content, motivation, and interpersonal attitudes are typical of personality disorder. As noted, inner polarization into contradictory "either or" affects and images self- and others makes these individuals prone to oscillate between periods of optimistic exaltation (reflecting idealization of new people and the associated self-image) and disillusionment (reflecting anger and devaluation of themselves and others, including those previously idealized), owing to failures in maintaining one's own unrealistic self-image. The unpredictable mood turnarounds are difficult to understand, as they seem to appear from nowhere, driven as they are by idiosyncratic internal agenda rather than by anything appreciably based in reality. One exception to the described mood instability is the stable low-key mood of the schizoid variant. Here, emotional detachment serves as a compensatory solution to the core fragmentation (discussed later) thereby reducing the importance of the interpersonal stage to maintain one's self-image, which is mostly supported unilaterally, in fantasy (interpersonal stage is otherwise vitally important in other variants).

One should keep in mind that people process sensory and factual information, including social clues, through a highly personal prism, basically a set of unique expectations reflecting one's unique personality organization. In personality disorder, the patient's expectations are extremized in order to match the unrealistic self-image. Such "tunnel-vision" communication makes these individuals prone to fail own expectations. As the most intense emotions are triggered by failures, they manifest negative emotions (dysphoria and anger in particular) more frequently and more intensely than positive emotions (elation), although the latter can be rather impressive as well.

\section{Core dysphoria: the default mood of personality disorder}

Dysphoria refers to a variety of chronic and pervasive but ill-defined negative moods common in all variants of personality disorder: emotional distress, emptiness and boredom, resentful neediness and dependency, sense of mental fragility, inadequacy, irritability, and deeply rooted sense of abandonment, among others.

\footnotetext{
${ }^{10}$ Affect is defined as a nonconscious, short-lived and usually intense psycho-physiologic brain state consisting of subjective conscious experience (emotion, feeling), associated ideas and fantasies, and biologic and behavioral response patterns. The concept of emotion is interchangeable with feeling, both referring to a conscious experience of an affect state. Mood is defined as a pervasive and sustained emotion, spreading over the entire mind, including cognition, motivation, and behavior, over a relatively long period of time.
} 
Such miserly moods in turn fuel negative interpersonal attitudes, notably sarcasm, bitterness, resentment, cynicism, active and passive aggression, among many others. The term "core dysphoria" implies its roots in the fragmented personality core or, more precisely, in the exhausting and ultimately futile struggle to achieve a sustained inner stability (Pazzagli \& Monti 2000). To wit, core dysphoria reflects never ending failures of internal expectations. Neuroimaging studies suggest a causal association of personality fragmentation, suboptimal prefrontal network development and organization, and chronic low mood in personality disorder, justifying the term "core dysphoria": a dysfunctional frontal lobe was found to be associated with intrapsychic and interpersonal features indicative of profound personality pathology in young adult females with chronic dysphoria (Lyoo et al. 2002).

\section{Recurrent episodes of atypical depression}

The constructs of atypical depression and "borderline" (fragmented) personality overlap. In most cases, fragmented personality can be found are the root of atypical depression. An episode of atypical depression typically occurs after major failures, relationship breakups, disappointments, in short, it follows unmet expectations in the external environment. Atypical depression is more severe than core dysphoria, but the two may coexist (a variant of "dual depression"). Clinically, episodes of atypical depression are distinguished from typical depression based on (i) preserved reactivity to pleasurable stimuli (the patient responds to "good news"), reversed vegetative symptoms of (ii) increased appetite or weight gain and (iii) hypersomnia, (iv) heavy or leaden feelings in limbs, and, as the cardinal feature, (v) profound and chronic rejection sensitivity ${ }^{11}$ not limited to mood disturbance. Rejection or abandonment sensitivity refers to a chronic, intense, irrational and usually unjustified expectation of rejection and rapid overreaction to it. The phenomenon is believed to be rooted in the continuing need for selfobjects: the patient dreads being rejected by his or her selfobject as this threatens not only the integrity but also the survival of his or her own self (selfobjects define and maintain the patient's sense of self). The dynamics is indicative of an early developmental deficit and is highly suggestive of personality disorder.

\section{A rich assortment of trait anxieties ${ }^{12}$ characterize personality disorder}

Biological trait anxiety refers to stable anxieties associated with the heritably excessive temperament

\footnotetext{
${ }^{11}$ Rejection sensitivity and abandonment anxiety are for all practical purposes equivalent concepts

12 "Trait" anxiety refers to stable individual dispositions towards anxiety ("how the person typically is" - a longitudinal view) while "state" to anxietyrefers to episodic anxieties which arises from changes in internal and external conditions ("how the person currently is" - a cross- sectional view).
}

trait $^{13}$ of HA, most prominent in negativistic-dependent $^{14}$, paranoid, narcissistic, and anankastic variants. Functional trait anxieties are rooted in the fragmented mind: pervasive uneasiness about physical health (hypochondriasis) and psychological safety (disintegration anxiety), intense separation anxiety (resulting from unfinished individuation), anxiety concerned with authenticity of existence (existential anxiety), and unique abandonment anxiety, a component of the wider rejection sensitivity syndrome which also includes distrust in one's own ability, lack of self and object constancy, among other phenomena. Separation and abandonment anxiety are usually intertwined and common with all variants of personality disorder, excepting the schizoid variant. Individuals with personality disorder are "stuck" in the separation-individuation phase, having not achieved a full separation and even less so individuation, they show a peculiar intolerance of being alone.

Continual struggle to compensate for inner fragmentation stirs anxieties unique to personality disorder (referred to as "fragmentation anxieties"), regardless of the variant and even in the context of low biological anxiety, e.g., in maladapted individuals with heritably low or moderate HA. Fragmentation-related trait anxieties arise as a function of (i) continual danger of disintegration, which fuels doubts about own psychological safety (disintegration anxiety), or (ii) precarious cohesion of the compensatory self, which fuels doubts about one's own authenticity and purpose (existential anxiety). Both anxieties are likely unique to humans who, in developing self- awareness, opened the door to selfdoubt, thereby expanding the repertoire of fear-inducing stimuli to abstract threats and metaphysical questions of purpose and meaning.

The natural course of fragmentation anxieties is fluctuating, reflecting the fluctuating degree of compensation achieved by deviant functioning. Because of such waxing and waning course, fragmentation anxieties may be misdiagnosed as state anxiety, frequently misleading treatment away from the root cause of the problem. Fragmentation anxiety requires long- term reconstructive psychotherapy with only sporadic and short- term use of anxiolytics during periods of exacerbations. Such "comfort pharmacotherapy" is indicated because surges of fragmentation anxiety can lead to the formation of new symptoms, deepening of existing symptoms, or to regression.

Disintegration anxiety can be thought of as a psychological equivalent of the fear of dying. It includes a profound feeling of mental fragility experienced by the patient who is struggling to maintain a cohesive

\footnotetext{
${ }^{13}$ High HA or anxious temperament is clinically presented and diagnosed as generalized anxiety disorder (GAD).

${ }^{14}$ We combine the DSM passive aggressive and dependent subtypes into the negativistic- dependent variant of personality disorder, based on our clinical observations that submissiveness and passive aggression regularly co- occur in this particular variant.
} 
sense of self and thus feels psychologically unsafe. Kohut (1977) understood this type of anxiety as the patient's "dawning awareness" that the cohesion of his or her self is precarious and could easily disintegrate under adverse psychological circumstances. Similarly, Klein (1952) talks about the "fear of invasive malevolence" within paranoid-schizoid position ${ }^{15}$, which (the fear) is experienced as coming from the outside but ultimately deriving from the projection of the person's imminent annihilation fears causing such paranoia. A form of disintegration anxiety is hypochondriasis, aka "health worry anxiety". The person demands diagnostic workups and does not respond to reassuring findings. Hypochondriasis arises from the core problem of fragmentation generating a deeply rooted distrust in one's own health, here physical health. It is thus usually suggestive of personality disorder, particularly narcissistic, histrionic, antisocial, and paranoid.

Existential anxiety refers to one's dim but unmistakable awareness of own pretense ("as if") and is rooted in fragmented personality. This form of trait anxiety arises from the ultimate inability of individuals with personality disorder to develop an authentic sense of meaning and true calling, but rather live by the dictate of false motivations and assumptions inherent in the compensatory self-image. Such artificial existence feels like a fabrication without genuine authenticity, passion, and purpose. Most patients distantly recognize their "as if" personality which is suggestive of some, albeit only phenomenal, passive noticing of their own pretense (discussed earlier).

\section{Withdrawal from the social world as unilateral adaptation: emotional detachment and social disinterest}

Reserved and aloof emotions and social disinterest are occasional with paranoid, anankastic, and antisocialschizoid individuals, and are typical of the schizoid variant of personality disorder. The defining features of the latter are social disinterest (referring to a peculiar lack of desire for social contacts or sexual experiences), preference for a solitary lifestyle and activities ("loners"), indifference to praise or criticism, blunted emotions (negative and positive alike), excessive daydreaming (autistic fantasy), and excessive introspection (ruminative self-examination). In contrast to other variants, schizoid individuals rarely seek interpersonal conflicts, do not actively disturb the environment, stay isolated and keep to themselves, and rarely break laws or engage in risky behaviors. In contrast to other variants which vitally need the interpersonal stage to maintain own self-image, individuals with the schizoid variant need minimal interpersonal approval. They appear as if they have abandoned bilateral interactions with the society and have withdrawn into the autistic world of fantasy

\footnotetext{
15 Paranoid-schizoid position is equivalent to fragmented personality
}

and daydreaming, with full control over mental events, which is instrumental in organizing internal fragments (a form of unilateral adaptation).

The main trait vulnerability for the schizoid variant is low social RD (social disinterest and detachment), coupled with low NS (stoicism, low impulsivity), low HA (low anxiety, low fear of uncertainty), and low PE (indolence, lack of ambition). It is this composite of globally hypoactive temperament, coupled with personality fragmentation, and compensatory withdrawal into fantasy that we consider a bona fide schizoid personality disorder. ${ }^{16}$

\section{Cognitive distortions, dissociative phenomena, and psychotic symptoms}

Chronic and subpsychotic ("soft") cognitive distortions observed in personality disorder fall into two broad categories:

Symptoms of loose (undisciplined) cognitive functioning reflects a strong presence of the primary process, dominated by wishes, conflicts, affects, and fantasy (Auchincloss \& Samberg 2012). The phrase "strong presence" is used to draw contrast with omnipresent primary process in psychosis. The concept of primary process refers to nonlinear, often nonverbal thinking which employs concrete symbolization, condensation, and displacement, resulting in disregard for logical connections or contradictions (e.g., a fallacious attribution of causal relationships between actions and events). Individuals with personality disorder characterristically manifest limitations in reality testing both in terms of deficits in the subtle aspects of interpersonal functioning (Kernberg 2015), and in their sense of self, filled with fantasies only vaguely recognized as wishful. The approximately even mixture of primary and secondary process distinguishes personality disorder from neuroses, where the secondary process, based on logic, reality, and problem-solving, is dominant.

Symptoms of biased cognitive functioning arise from the unrealistic self-image which dictates variantspecific cognitive distortions and motivational priorities. These are made possible by the presence of the primary process. Biased cognition therefore can be thought of as a special case of loose cognition characterizing a particular variant of personality disorder. For illustration, egocentric perception of reality, whereby events are primarily defined through the prism of one's own wishful needs, is a pervasive cognitive distortion present in all variants but with a differing theme: misattribution of admiration by others of narcissistic personalities ("I am admired, therefore I am"), pervasive suspiciousness of paranoid personality ("I am persecuted, therefore I am"), sexualization of interpersonal contacts of histrionic personality ("I seduce, therefore I am"), alpha-type dominance of

\footnotetext{
${ }^{16}$ In contrast to schizoid features without personality disorder manifested by individuals within the "Schizophrenia spectrum".
} 
antisocial personality ("I intimidate, therefore I am"), or withdrawal from external reality and reliance on uncontested fantasy of schizoid personality ("I define who I am"), among others.

Individuals with any variant of personality disorder can more or less transiently regress to the psychotic level of mental functioning, especially those with a family history of psychosis (i.e., those genetically closer to the threshold for psychosis) and/or when under stress (hence the label "brief reactive" psychoses). The intensity of stress-related anger or paranoid fears may erode the precarious stability of the mind achieved through the compensatory deviant self. One may speculate that intense negative affects in the context of severe stress can break through the protective barrier erected by the splitting mechanism and thereby open the floodgates for the primary process to temporarily impair reality testing, with psychosis as a manifestation. These episodes are indeed of short duration, from several hours to several days, and are thus amenable to confrontation and, later in therapy, to interpretation.

\section{Anger and aggression: the default affect - behavior complex of personality disorder}

Kernberg (2015) provides an insightful analysis of ubiquitous anger and aggression in personality disorder: massive negative representations make the individual predisposed toward experiencing others as persecutory and/ or rejecting. The experience is easily activated by negative stimulation in interactions, which puts the patient in a chronically assumed role of a victim. Such a fixed persecutor-victim relationship leads to anger and aggression which the patient, by the mechanism of projective identification, attributes to others. In order to maintain the omnipotent power position and sense of control, the patient (victim) seeks conflicts with others (persecutors), and eventually he or she (the patient) becomes the victimizer. This dynamics makes individuals with fragmented personality prone to aggression, usually impulsive-affective interpersonal conflicts ${ }^{17}$ in the context of (mis)perceived threats to one's compensatory but precarious self-image. This pattern explains the presence of aggression in all clinical variants of personality disorder, fearful ${ }^{18}$, impulsive, and aloof alike. The act of impulsive aggression is not gain oriented but is an end unto itself, aimed to destroy or correct the perceived provocation. Violence is generally rare excepting the antisocial variant, but even here not invariable

Predatory aggression is rare with personality disorder. Poor executive function, erratic moods, and un-

\footnotetext{
${ }^{17}$ Here, aggression does not denote a heritably high biological disposition, but rather arises from the described dynamics of personality disorder. Aggression as a heritable disposition, related to low frustration tolerance and impulsivity associated with excessive NS, acts as early trait vulnerability for antisocial and histrionic variants of personality disorder.

${ }^{18}$ Passive-aggression in the negativistic-dependent variant.
}

stable personal goals are not the best set of features for successful predation. The latter requires effective impulse control and executive function, both a function of cognitive networks, with less involvement of the hypothalamus and limbic structures. The predator exhibits patience and careful planning to disguise his or her intentions, choose a vulnerable target, and strategize the attack, which is typically not a response to an immediate threat or provocation but is driven by the expectation of gain.

\section{Character disorder: a useful but abandoned concept}

The concept of "character disorder" captures the pathology of social behavior, including career predation, which is not associated with the described neural and mental pathology of personality disorder. Rather, career predation reflects one's adaptive strategy by choice, whether intuitive or deliberate, usually made in adolescence or early adulthood. To refine public, forensic, and scientific understanding of antisociality and criminality, we advocate that the concept of "character disorder" be reinstated as a diagnosis ${ }^{19}$. The concept captures not only career predators, as discussed, but also a spectrum of other egotistic, repulsive, illegal, and/or obnoxious social behaviors (arrogance, theft, entitlement, greed) in the context of statistically integrated personality but corruptible character and morality by choice, i.e., intuitive or deliberate strategy to obtain resources and/or get ahead at the expense of others.

\section{Interpersonal relations: conflict as the only resource to maintain the sense of self}

Personality disorder is experienced intrapersonally, expressed interpersonally, and diagnosed in both contexts. Accordingly, DSM-5 and ICD-11 place diagnostic emphasis on both self-pathology and interpersonal relations. As noted, an adaptively suboptimal mind, as is the case with personality disorder, continues to need sources of organization, both internally (through fantasy) and interpersonally (through a continual need for selfobjects who define and maintain the sense of self). Interpersonal conflicts are actively sought to change others' reactions to one's own maladaptive style or to coerce them into following the expected exposition supplied by the supporting cast of selfobjects (alloplastic adaptation). The "provocateur" is important for the psychological stability of the provoked, who thus cannot just avoid confrontation, but instead feels compelled to re-establish the image through the conflict. Such hostile interpersonal conflicts further cement the polarization of the inner world and thereby perpetuate the core psychopathology of all variants, excepting the schizoid variant where such conflicts are rare.

\footnotetext{
19 The term and concept have been abandoned by the DSM and ICD systems.
} 
Interpersonal stage is where the deviant compensatory self finds its materialization (fantasy is another option, but rarely sufficient, excepting the schizoid variant). The dependence on interpersonal relations to maintain the deviant self-image may explain why traitaggression sometimes alternates with helpless dependency: the dynamics reflects a frustrating stalemate between the vital need for inner organization and one's inability to achieve it without the supporting roles of selfobjects i.e., others. Each variant of personality disorder demonstrates its own specific style of social deviance, the patient's common style of interaction as the means to maintain inner organization. These variantspecific styles define interpersonal contacts. Individuals with the schizoid, paranoid, and antisocial variant of personality disorder are characterized by social coldness and disinterest, lack of empathy, low intimacy, and occasional predatory crime (antisocial and paranoid variants are more vindictive and domineering than the schizoid variant). With exhibitionistic and intrusive variants (antisocial, narcissistic, histrionic), the distinguishing features are grandiosity and social coldness (narcissistic and antisocial), or sexualization of all relationships, theatricality, and rush into intimacy (histrionic). Reticence, rigidity, and perfectionism characterize interpersonal relationships of the anankastic variant, while passive-aggression combined with dependency characterize the negativistic-dependent variant. As noted, the shared affect state for all variants is resentful anger of a victim manifested as fantasies of omnipotent control of others and frequently as overt aggression (one exception are the idealized selfobjects, although this can quickly change). Each variant shows a significant association with vindictiveness, social distrust and suspiciousness, and a decreased concern about the needs of others. All of these features are accompanied by minimal personal distress, suggestive of ego-syntonicism. All variants are also characterized by opportunistic, corruptible, and non-abstracted morality in interpersonal relationships, usually resulting in minor transgressions and manipulation, only exceptionally in major violent crimes or predation, as discussed. Finally, all variants manifest character traits of social intolerance, egocentricity, selfishness, greed, etc., because character development was primarily guided by the mandate for internal organization, not to maximize social adaptation, as is normally the case.

\section{SYMPTOM DOMAIN \\ BASED TREATMENT OF PERSONALITY DISORDER}

For the majority of the described symptoms of personality disorder the most effective treatment is reconstructive in-depth psychotherapy which targets the core problem through corrective emotional experience. Lieb et al. (2010) review drug trials of "borderline" personality and conclude that medications may be effective in treating turbulent affects and behaviors but not the overall severity of the disorder. However, we agree with Blais et al. (2016) that "modest empirical evidence and considerable clinical benefits" continue to support the use of medications in the overall treatment of personality disorder. Symptom-based pharmacological suppression of affects and impulses helps clear the way for corrective emotional experience in psychotherapy and also helps suppress disruptive affects in real life, an important early clinical goal.

\section{The symptomatic architecture of personality disorder: four main symptom domains}

In symptom-based approach, symptoms are targeted outside of their neural and mental mechanisms: dysphoria with antidepressants, unstable mood with mood stabilizers, and so on. The rationale for drug choice is based on analogy with treatments proven effective for "major" syndromes (depression, mania, anxiety and psychosis), rather than a theory driven approach specific for personality disorder. This lack of specific hypothesis about underlying mechanisms to guide treatment is the fundamental difference between mechanism-based and symptom-based pharmacotherapy of personality disorder. Both approaches are bottom-up, as both focus on heightened subcortical affect reactivity, but they differ in another important way: symptom-based approach suppresses the pathological equilibrium in which affects dominate cognition, while mechanism-based approach aims to activate homeostatic equilibration of a specific trait vulnerability, which now can be integrated into the mind in a natural way (Svrakic et al. in prep.). However, symptom-based approach may be closer to the root psychobiology of personality disorder than one may think. The four empirically derived symptom domains (see below) not only correspond to the latent genotypic-phenotypic architecture of personality disorder (Livesley et al. 1998) but also to the high (extreme) ends of normal temperament traits (Cloninger et al. 1993), the latter are established as trait vulnerabilities for personality disorder (Svrakic et al. 1993). The high correspondence between observable symptoms arranged into domains, latent phenotypic-genotypic dimensions, and normal albeit excessive temperament traits narrows, but does not close the gap between symptom-based and mechanism-based approaches, as indicated by the non-selectivity of symptomatic pharmacotherapy which affects multiple symptom domains simultaneously (discussed below).

The symptom-domain pharmacotherapy targets phenomenology and symptoms that are not readily recognizable to be typical of individual variants of personality disorder, e.g., in DSM-5 criteria (APA 2013). This is because the symptom-based approach is more effective in suppressing symptoms of shared fragmentation (such as mood instability, core dysphoria, trait anxiety, anger and aggression, etc.) than those of variant specific behavior styles (narcissistic, histrionic, dependent, etc). Simply put, no available medication is capable of treating 
narcissistic or histrionic attitudes, as these are compensatory epiphenomena, emerging mental properties which organize the fragmented mind. These variant-specific symptoms are not suitable as targets for pharmacotherapy. The latter is most effectively organized around four empirically derived domains of symptoms, each rooted in personality fragmentation and thus each shared by all variants to a variable degree, although with some predilection for certain variants (e.g., impulsivity is most impressive in histrionic, antisocial, and narcissistic variants).
The four empirically derived symptom domains are:

- Mood and anxiety dysregulation (chronically unstable mood, core dysphoria, recurrent atypical depression, chronified anxieties);

- Behavior dysregulation (interpersonal affective aggression, poor impulse control);

- Emotional detachment (social disinterest, low intimacy, lack of empathy, restricted expression of emotion);

- Cognitive dysregulation (loose and biased cognition, transient psychoses).

Table 1. Pharmacotherapy of four target symptom domains of personality disorder

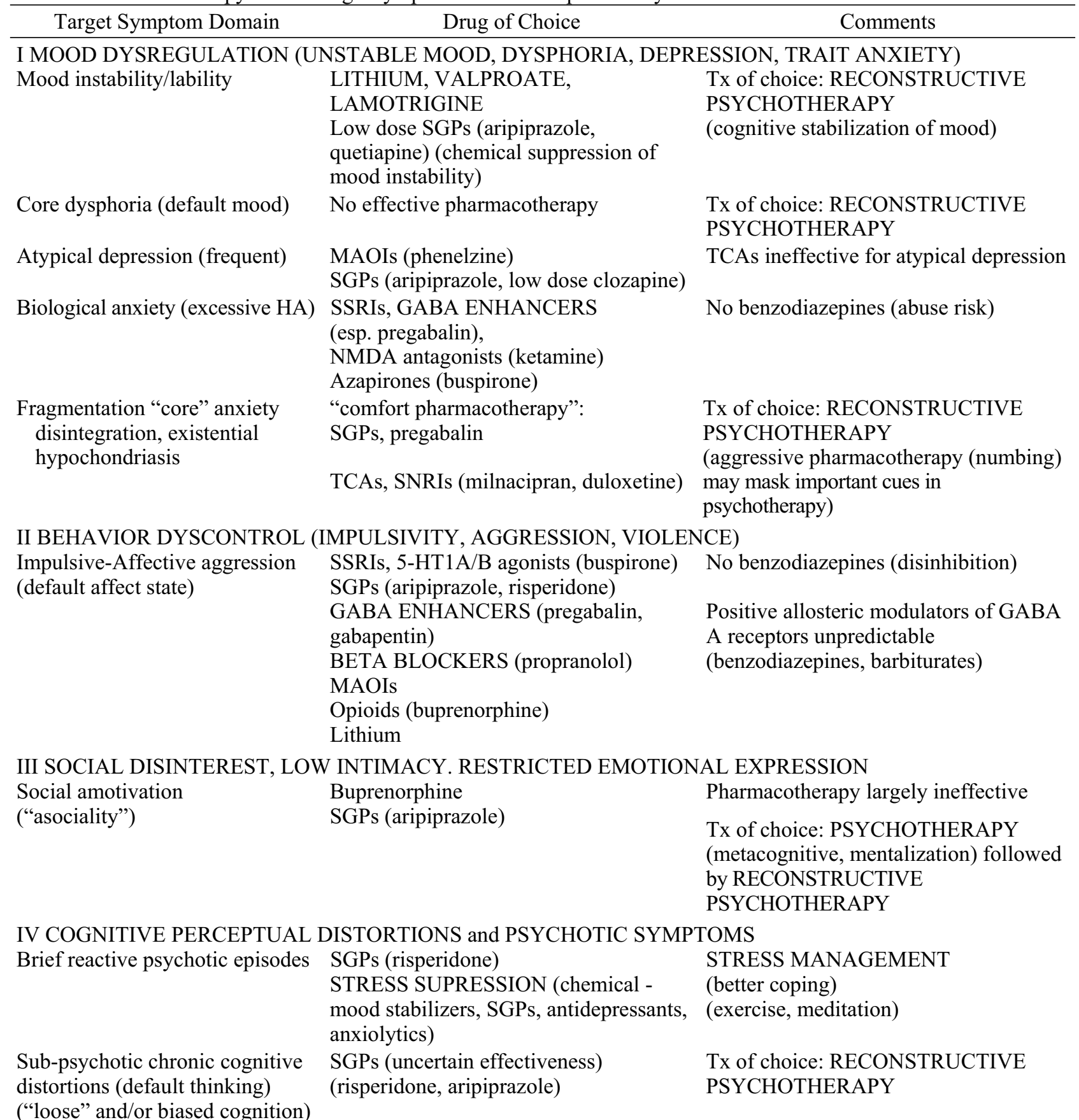
("loose" and/or biased cognition)

Legend: CAPITAL LETTERS - FIRST LINE and MAJOR INDICATION; lower case letters - second line; SGPs - second generation psychotropics; BNZD - benzodiazepines; SSRI - selective serotonin reuptake inhibitors; SNRI - serotonin norepinephrine reuptake inhibitors; GABA - gamma aminobutyric acid; MAOI - monoamine oxidase inhibitors 


\section{Pharmacotherapy of symptom domains: non-selective but clinically useful}

Pharmacotherapy targeting the above four domains is non-selective, as medications affect multiple domains simultaneously. For example, second generation psychotropics (SGPs) ${ }^{20}$ are used for all four symptom domains (e.g., mood and anxiety dysregulation, behavior dysregulation (aggression), emotional disinterest, and cognitive symptoms of psychosis). Similarly, serotonergic antidepressants are the treatment of choice for mood, anxiety, and aggression domains. Such nonselectivity may reflect a number of factors, the most likely being that the four domains are heterogeneous entities, co-regulated by anatomically overlapping, neuronally intertwined, and functionally interrelated brain networks involved in mood, motivation, and cognition. Recommendations for pharmacotherapy targeting symptom domains are summarized in Table 1.

The recommendations listed in Table 1 are made based on the available, albeit rather limited literature as well as on the authors' clinical experience. The evidence for the recommendations is generally weak, this reflecting lack of good clinical studies rather than negative findings. Table 1 is self-explanatory. Thus, we have chosen to address a few selected topics that might be of interest to the reader.

\section{Symptom domain I: Mood Dysregulation and Anxiety Syndromes}

This symptom domain includes mood instability, core dysphoria, recurrent atypical depressions, episodes of major depression, and a spectrum of trait anxieties. Based on Livesley et al. (1998), we include trait anxiety in the mood dysregulation domain.

Although non-bipolar in nature, mood instability of fragmented personality is treated by traditional mood stabilizers, lithium, valproate, and lamotrigine in particular (the latter slightly preferred by most experts). As second-line, low to moderate doses of atypical SGPs stabilize mood by unknown but likely diverse mechanisms. All have been approved by Food and Drug Administration (FDA) for bipolar disorder and all show modest effectiveness in suppressing mood instability fueled by the specific dynamisms of personality disorder as well ("chemical suppression"). One explanation for such unexpected but welocme effectiveness is that all moods, whether biological, internally generated, or reactive by nature, ultimately materialize within their respective neurobiological circuits and become conscious by the work of the same general network of cognition. Here, pharmacotherapy affects both cortical and subcortical circuitry in a global pattern, by general slowing of neuronal activity (lithium, valproate), enhancing inhibitory GABA activity (valproate), or suppressing activating glutamatergic mechanisms (lamotrigine). SGPs are likely

\footnotetext{
${ }^{20}$ The term "psychotropics" is preferred over "antipsychotics" for these medications, as they are commonly used in nonpsychotic syndromes, such as anxiety and affective disorders.
}

to benefit unstable moods via serotonergic and dopaminergic effects, as both of these brain systems are relevant in mood and anxiety symptoms. Reconstructive in-depth psychotherapy is more specific for the mood instability of personality disorder and is thus the treatment of choice ("mental stabilization").

\section{Symptomatic management of low moods of personality disorder}

Core dysphoria is the default mood of fragmented personality, and is notoriously refractory to pharmacotherapy. Some options are presented in Table 1. Corrective emotional experience ("re-parenting") in expert psychotherapy helps both the dysphoric mood and the co-occurring features indicative of fragmentation, such as rejection sensitivity or clinging dependency (Beatson \& Rao 2012).

Any form of episodic depression comorbid with personality disorder, whether typical or atypical, is more resistant to traditional antidepressants and electroconvulsive therapy (ECT), than depression in individuals with no personality disorder. Atypical depression is believed to be biologically distinct from major depression because of its inferior response to standard antidepressants and ECT, and superior response to phenelzine, a MAOI. For phenelzine nonresponders, instead of switching to a non-MAOI antidepressant, augmentation with or switching to one of the SGPs may be more effective, although this has not been studied systematically.

Given its frequent comorbidity with major depression, some have proposed that borderline (fregmented) personality merely represents an unusual manifestation of unipolar depression. As noted earlier, the most compelling evidence against such spectrum relationship is that treatment of major depression does not improve core borderline symptoms, while psychotherapy of borderline personality tends improve both personality and depression (reviewed in Beatson \& Rao 2012).

\section{Prozac "poop out": is it time to use pulse treatments for the phasic illness of depression?}

The recent increase in treatment refractory depression, now estimated at about $30 \%$ of all cases, coincides with the expanding use of selective serotonin reuptake inhibitors - SSRIs, usually after a good initial response (the phenomenon has been dubbed "Prozac poop-out" or "tachyphylaxis"). Dual reuptake inhibitors (tryciclic antidepressans - TCAs and serotonin-norepinephrine reuptake inhibitors - SNRIs) appear to have lower rates of progressive decrease in response (Posternak \& Zimmerman 2005). Tachyphylaxis is hypothesized to reflect pharmacodynamic tolerance (desensitized receptors), pharmacokinetic tolerance (decreased quantity of medication reaching the target site), or new onset apathy, all documented with SSRIs.

The increase in treatment resistance possibly related to SSRIs has led many to question the typically long 
treatment duration, frequently lasting years and even decades. Criteria for discontinuation of antidepressants are vague. Clinicians are reluctant to discontinue antidepressants to avoid relapse, a real risk for many patients. However, a significant number of patients taking antidepressants relapse anyway (cca. $20-44 \%$ of patients on SSRIs, lower for TCAs), so the relapse rates cannot be much worse with discontinuation. A few logical strategies to reduce the incidence of refractory depression are listed below. We emphasize that none has been supported by systematic research. One possibility is to treat depression as a phasic illness in a phasic manner (aka "pulse treatment") with SSRIs. Drawing from our concept of harnessing homeostasis for healing ${ }^{21}$, drug holidays during remissions may help preserve the brain's ability to activate its homeostatic response in case SSRIs are needed again. Mood stabilizers (lamotrigine in particular) may reduce the risk of relapse during the SSRI holidays. Another strategy is to alternate between SSRIs, NRIs, and SGPs at regular intervals, which would mean even switching patients who are doing well on a certain drug.

\section{Comfort pharmacotherapy for trait anxieties}

As discussed, a rich variety of trait anxieties is observed with personality disorder, from biologically anxious temperaments (heritably high HA), to a spectrum of anxieties rooted in the fragmented personality core, such as disintegration anxiety and hypochondriasis, existential anxiety, and abandonment anxiety. Heritably high HA is effectively treated with SSRIs, GABAenhancers - especially pregabalin ${ }^{22}$, mild NMDA antagonists (ketamine), and buspirone (a partial 5-HT1A receptor agonist). Treatment of choice for fragmentationrelated trait anxieties is reconstructive in-depth psychotherapy. Pharmacological numbing of these trait anxieties does not address the root cause, and in fact could mask important cues in psychotherapy. Medication is thus not used beyond the minimum amount and time necessary to ensure some comfort ("comfort pharmacotherapy"). Antidepressants are not very effective for hypochondriasis, but TCAs may be more effective than SSRIs, although the latter have not been studied as frequently.

\footnotetext{
${ }^{21}$ Pharmacotherapy can be designed to activate corrective restoration of homeostasis to optimize all relevant participants in a complex trait. The class of SSRIs provide example of such on demand homeostatic recalibration: the initial increase in extracellular serotonin, a mechanistically fundamental molecule in anxious temperaments, triggers weeks of homeostatic neuroadaptations which eventually downregulate the central serotonergic system. This is widely believed to be responsible for the SSRIs efficacy in depression and anxiety states, now both believed to be "hyper-serotonergic".

${ }^{22}$ Pregabalin has been approved in Europe for GAD, a clinical name for heritably high HA without personality disorder.
}

\section{Symptom domain II: Dysregulation of Behavior (Aggression, Impulsivity, Violence)}

Anger and aggression are default affect-behavior traits in personality disorder and perpetuate its psychopathology. As discussed, chronic interpersonal aggression occurs as a reaction to misperceived threats to one's own compensatory but precarious self-image. Here, we limit our discussion to impulsive affective aggression, by far the most frequent form of aggression in personality disorder. The neural mechanisms of affective aggression are reviewed to lay the groundwork for pharmacological considerations.

\section{The neuroanatomy of human aggression}

Two networks, one primarily subcortical (aggression as instinct), the other primarily cortical (aggression as decision), both intersecting in the amygdala, are postulated to underlie the complex phenomenon of human aggression (Victoroff 2017). The cortical network (involving the ventromedial, orbitofrontal, dorsolateral PFCx, the anterior cingulate cortex, with the key nexus in the amygdala) regulates aggression as a behavior assessed to be the only resource in the given context (e.g., protecting one's child against criminal abduction in progress). This is a partly conscious process capable of either suppressing automatic amygdala reactions or to proceed with aggression if deemed necessary. The subcortical network regulates nonconscious, instinctive aggression in emergencies, e.g., a reflex reaction towards the home intruder who threatens the homeowner's life. The subcortical circuits of aggression involve the hypothalamus, the nucleus accumbens/septal region, the dopaminergic ventral tegmentum, the serotonergic raphe nuclei, and the dorsal periaqueductal grey (PAG), again with the key nexus in the amygdala (Victoroff 2017). The same subcortical circuitry also regulates a number of other instinctive social behaviors (mating, dominance, etc.) and is known as "subcortical social behavior network" in animals (Newman 1999). In personality disorder, both of these loops contribute to trait aggression. First, the hypofunctional PFCX renders subeffective cortical feedback regulation of affect-driven behaviors. Second, most variants of personality disorder have a heritably heightened fear and anger reactivity, both of which predispose them to aggression via subcortical activation. The neuroanatomy and neurophysiology of aggression are complex (which is understandable given its evolutionary significance), making pharmacotherapy of aggression equally complex.

\section{The neurophysiology of impulsive aggression: serotonin (5-HT) deficiency at the root of escalated aggression ${ }^{23}$}

We summarize the vast literature on the neurophysiology of escalated affective aggression as follows: phasic spikes of 5-HT release form the dorsal raphe nuclei to the

\footnotetext{
${ }^{23}$ Serotonin deficiency does not play a role in adaptive aggression (i.e., aggression within the norms for a species).
} 
medial PFCx are believed to contribute to the initiation and maintenance of aggressive outbursts in social conflicts (Takahashi et al. 2015). Such bursts in 5-HT activity in individuals prone to escalated aggression lead to low basal 5-HT neurotransmission, a neuroadaptive downregulation in habitual aggressors (this is the basis for the well-known "serotonin deficiency" hypothesis of aggression). The 5-HT deficiency in turn compromises serotonergic regulation of the cortical glutamatergic neurons and their feedback to the limbic system and midbrain/ brainstem nuclei, notably the dorsal raphe nuclei, the ventral tegmentum, and the locus-coeruleus, affecting in the process the serotonergic, dopaminergic, and noradrenergic modulation of aggression. This results in, respectively: i) more phasic bursts in 5-HT release to the medial PFCx, ii) dopaminergic potentiation, permission, and reinforcing of victorious aggressive encounters, and iii) noradrenergic potentiation of aggression at the level of hypothalamus and PAG. As a general rule, glutamate may function to exaggerate the excitability of the neural players involved in aggressive behavior, particularly when aggression is intense, i.e., glutamate may sensitize an individual to become more aggressive (Miczek \& Fish 2005). GABA, by contrast, generally inhibits aggression, as do opioids and serotonin.

\section{Pharmacotherapy of affective aggression}

With the above in mind, SSRIs and GABA-enhancers $^{24}$ are first line treatment of affective aggression. In addition to SSRIs, a number of other drugs suppress the phasic spikes in 5-HT release (necessary for the initiation of aggression as noted), most notably the SGPs which have both 5-HT2A antagonism and 5-HT1A partial agonism (aripiprazole, risperidone) and 5-HT1A and 5-HT1B autoreceptors agonists (buspirone in particular). The brain's noradrenergic system may have a permissive role in aggressive behavior, facilitating fight over flight in response to a challenge, perhaps at the level of the hypothalamus (reviewed in Siegel \& Victoroff 2009). Hence, noradrenergic blockers ("beta-blockers") may suppress such potentiation of aggression.

The dysregulated accumbal and cortical dopamine, reflecting the PFCx overactivation of the ventral tegmentum, the source of dopamine for the meso- limbiccortical pathway, may play a role in the incentive salience but not in the significant modulation of offensive aggression (Miczek \& Fish 2005). The activated ventral tegmentum also projects to the medial hypothalamus and other limbic structures, which may facilitate both offensive and defensive rage. Not much evidence exists for the efficacy of dopaminergic receptor blockers in suppressing the incentive salience of and/or in revoking permission for aggression.

\footnotetext{
${ }^{24}$ GABA-enhancers increase extracellular GABA, while positive allosteric modulators change the conformation of GABA A receptors (benzodiazepines, barbiturates, alcohol) and have unreliable effects on aggression.
}

Polymorphisms of the monoamine oxidase A (MAO A) gene appear to facilitate affective aggression by modulating the structure and function of synaptic glutamatergic NMDA receptors in the PFCx, ultimately affecting cortical control of aggression. Clinically, MAO inhibitors can be useful in affective aggression triggered by provocation, although the result may in part may be a function of improved atypical depression.

Finally, as mentioned, opiates have a serenic effect. Suppression of defensive rage is believed to be mediated via $\mu$-opioid receptors in the PAG. This mechanism opens the door, at least theoretically, for partial $\mu$ receptor agonists, like buprenorphine, to be tried in human affective aggression.

Multiple double-blind trials have shown the efficacy of lithium salts in the treatment of affective aggression at therapeutic levels for mania. Similar efficacy has been reported for combinations of lithium either with SGPs or with many anticonvulsants (reviewed in Comai et al. 2012b). The exact nature of lithium's anti-aggression effectiveness has not been clarified. It is likely multifaceted (reviewed in Beaulieu \& Caron 2008, Pasquali et al. 2010). Lithium's cellular effects on second messanger pathways that modulate neuronal excitability, a possible antimanic mechanism, may also stabilize affective aggressive outbursts and, as discussed, mood instability. There is some evidence that, in a chronic treatment paradigm, lithium upregulates glutamate reuptake and decreases glutamate release, another possible antimanic mechanism (reviewed in Hahn et al. 2004) which may also help the individual to control escalated aggression.

\section{Symptom domain III: Social Disinterest, Low Intimacy, Restricted Emotional Expression}

Emotional detachment, reserved and aloof emotions, emotional poverty, and disinterest in social relations are most frequently observed with schizoid, paranoid, anankastic, and antisocial individuals the latter with prominent schizoid traits. To our knowledge, no data exist for pharmacotherapy of the globally hypoactive temperament of schizoid personalities. Clozapine has been shown to alleviate negative symptoms in with Schizophrenia, where social withdrawal reflects "primary asociality" with specific neural correlates (Kaiser et al. 2016). It is not clear whether clozapine, or any other drug, may affect the secondary asociality of schizoid personality, here a compensatory maladaptive style. Aripiprazole, a ligand-biased partial D2 and D3 agonist ("smart drug") with documented benefits for schizotypal symptoms but, to our knowledge, no studies in schizoid personality disorder may be tried (here mechanism-based and symptom-based approach overlap) ${ }^{25}$.

\footnotetext{
25 Mechanism-based treatment of low social RD includes oxytocin, serotonin-norepinephrine reuptake inhibitors - SNRIs (desvenlafaxine, milnacipran, levo-milnacipran), norepinephrine reuptake inhibitors -NRIs (atomoxetine), MAOIs, psychostimulants, and opioids (buprenorphine); (Svrakic et al. in prep.).
} 
Metacognitive $^{26}$ and mentalization based psychotherapy aiming to enhance one's appreciation for his or her own and other people's mental dynamisms are also indicated for individuals with schizoid personality disorder, mainly as the groundwork for reconstructive psychotherapy of fragmentation.

\section{Symptom domain IV: Cognitive-Perceptual Distortion and Psychotic Symptoms}

As discussed, this domain includes brief reactive psychoses as well as "soft" cognitive distortions, notably loose and biased cognition. Symptom-based medications are listed in Table 1. Risperidone is the most frequently used agent. Stress reduction is highly important as brief reactive psychoses typically occur in the context of acute stress or personal crisis. Experience of stress can be reduced by medication ("chemical suppression"), such as mood stabilizers (esp. valproate), antidepressants (e.g., mirtazapine), SGPs (aripirazole), GABAenhancing anxiolytics (pregabalin), or positive allosteric GABA A modulators (benzodiazepines). For example, alprazolam was shown to improve paranoia in borderline individuals, presumably due to anxiolytic anti-stress effects. Short-term use of benzodiazepines for personality disorder increases the risk of behavior disinhibition and aggression, while long-term use increases the risk of overuse. If prescribed, benzodiazepines should be monitored for abuse and used only short term until the reactive psychosis is resolved. Alternative approaches to reduce stress include better coping skills, achieved in psychotherapy and meditation.

\section{FINAL COMMENT}

\section{The second generation atypicals have transformed pharmacotherapy of non-psychotic syndromes}

Drawing from the dopamine hypothesis of psychosis, the group of first generation antipsychotics (aka "typicals") evolved from low-potency, sedating compounds in the 1950s (e.g., chlorpromazine) to potent D2 antagonists in the 1970s (e.g., haloperidol). These drugs are primarily used to treat the positive symptoms of psychosis, such as delusions and hallucinations. Administered to nonpsychotic individuals with personality or mood problems, typical antipsychotics have no desired specific effects but a plethora of undesired effects, including irreversible tardive dyskinesia.

A number of second generation agents - SGPs (aka "atypicals") have been developed with multiple modes of pharmacodynamic actions, primarily targeting but not limited to DA and 5-HT neurotransmission. SGPs cause less sedation and have a much more favorable side-

\footnotetext{
${ }^{26}$ Metacognition refers to the human capacity to be aware of and control one's own thoughts and internal mental processes.
}

effect profile in comparison with typical antipsychotics. In addition to antipsychotic effects, they also have impressive antidepressant, antimanic, mood- stabilizing, and anxiolytic effects, likely reflecting multiple modes of action and/ or functional overlap among brain networks involved in mood and cognition.

\section{Clozapine and aripiprazole stand out in the group of atypicals}

Clozapine, to date the most effective medication for positive, negative, and disorganization symptoms of Schizophrenia, is being increasingly used off label, to treat refractory depression, atypical depression, bipolar disorder, anorexia, severe anxiety disorders, and personality disorder, among others. The complex pharmacodynamic profile of clozapine may in part explain such wide spectrum effectiveness, which could be related to either multiple specific mechanisms or a combination of those. In addition to serotonergic (5- HT2A, 5- HT1A) and dopaminergic antagonism (mostly at D4 receptors), clozapine is also a histone deacetylase (HDAC) inhibitor, thereby mediating epigenetic increase in GABA production and GABA modulation of cortical pyramidal neurons, an effect believed to improve cognitive processing and top-down cortical control of subcortical functions. Also, clozapine is believed to stimulate the synthesis of GABAergic neurosteroids, an effect that may explain its efficacy not only in psychosis but also in chronic anxiety and vulnerability to stress. By yet another mechanism, clozapine blocks glycine transport, an effect that may enhance NMDA neurotransmission (Javitt et al. 2005) and perhaps facilitate neuroplasticity and new learning. In non-psychotic syndromes, clozapine is used at low dose (25-100 mg/day, BID dosing), while its plasma levels are kept within the low-to-medium range $(100-250 \mathrm{ng} / \mathrm{mL})^{27}$. Clozapine is highly protein bound, hence plasma levels are monitored to ensure its presence in the plasma in its free, active form (the levels are used to guide the dosing). Clozapine norclozapine ratio is kept at 2:1 at least, to avoid the cardiac and hematologic side effects of norclozapine ${ }^{28}$.

Aripiprazole, originally marketed as an antipsychotic, is now more frequently used for other indications, such as bipolar disorder, major depression, dysthymia, impulsive aggression, and trait anxiety in individuals with and without personality disorder, either as monotherapy or as an augmentation strategy. Aripiprazole is frequently referred to as a "third- generation" psychotropic to illustrate its unique action profile which has been dubbed a "biased ligand" or "smart" pharmacological agent working at D2 and possibly D3 dopaminergic

\footnotetext{
${ }^{27}$ Higher levels cca. $300-400 \mathrm{ng} / \mathrm{ml}$ are required to treat psychosis

${ }^{28}$ Many undesired effects can be minimized by careful clinical and laboratory monitoring
} 
receptors. It can act as a full antagonist, partial antagonist, and partial agonist at D2/D3 receptors. The type of action is determined by local DA levels, cell type, and functional and signaling status of the target cells. Aripiprazole is also a partial 5- HT1A agonist and a full 5- HT2A antagonist, and it has NMDA, histaminic, and adrenergic activity. The action profile also includes multiple cellular pathways within several cortical and subcortical neurotransmitter circuitries as well as modulation of gene expression distinct from other antipsychotics (reviewed in de Bartolomeis et al. 2015). Given its superb efficacy in depression, four- and sixweek injectable aripiprazole can be thought of as the first long- acting injectable antidepressant.

Despite relative safety, caution is advised. The decision to use SGPs long term requires consideration of potential undesired effects and should be made with the informed consent of the patient. Body weight and blood sugar must be closely monitored, so that early signs of metabolic and other adverse effects are recognized and prevented.

\section{Acknowledgements:}

Some of this material was originally published in "The Fragmented Personality: An Integrative, Dynamic, and Personalized Approach to Personality Disorder" by Dragan Svrakic \& Mirjana Divac-Jovanovic, and has been used by permission of Oxford University Press https://global.oup.com/academic/product/the-fragmentedpersonality- 9780190884611 ?q=the $\% 20$ fragmented $\%$ 20Personality\&lang $=e n \& c c=u s$

Conflict of interest: None to declare.

\section{Contribution of individual authors:}

Dragan Svrakic designed the paper.

Mirjana Divac-Jovanovic \& Naazia Azhar contributed to the write up and the final appearance of the paper.

\section{References}

1. American Psychiatric Association: DSM-5. APA Press, Washington DC, 2013

2. Auchincloss E, Samberg E: Psychoanalytic terms and concepts. American Psychoanalytic Association and Yale University Press, New Haven and London, 2012

3. Beatson JA, Rao S: Depression and borderline personality disorder. The Medical Journal of Australia 2012; 1(Suppl 4):24- 27

4. Beaulieu JM, Caron MG: Looking at lithium: Molecular moods and complex behavior. Molecular Interventions 2008; 8:230-241

5. Blais MA, Smallwood P, Groves JE, et al.: Personality and personality disorders. In Stern T, Fava M, Wilens TE, Rosenbaum (eds.), Massachusetts General Hospital Comprehensive Clinical Psychiatry, 433-444. Elsevier, New York, 2016
6. Comai S, Tau M, Pavlovic Z, et al.: The psychopharmacology of aggressive behavior: A translational approach part 2: Clinical studies using atypical antipsychotics, anticonvulsants, and lithium. Journal of Clinical Psychopharmacology 2012b; 32:237-260

7. de Bartolomeis A, Tomasetti C, Iasevoli F: Update on the mechanism of action of aripiprazole: Translational insights into antipsychotic strategies beyond dopamine receptor antagonism. CNS Drugs 2015; 29:773-799

8. Cloninger CR, Svrakic DM, Przybeck TR: A psychobiological model of temperament and character. Archives of General Psychiatry 1993; 50:975-990

9. Divac-Jovanovic $M$, Svrakic DM, Lecic-Tosevski D: Personality disorders: model for conceptual approach and classification, part I: General model. American Journal of Psychotherapy 1993; 47:558-571

10. Gratton C, Laumann TO, Nielsen AN, et al.: Functional brain networks are dominated by stable group and individual factors, not cognitive or daily variation. Neuron 2018; 98:439-452

11. Hahn CG, Gyulai L, Baldassano CF et al.: The current understanding of lamotrigine as a mood stabilizer. Journal of Clinical Psychiatry 2004; 65:791-804

12. Javitt DC, Duncan L, Balla A et al.: Inhibition of system $A$ - mediated glycine transport in cortical synaptosomes by therapeutic concentrations of clozapine: implications for mechanisms of action. Molecular Psychiatry 2005; 10:276-286

13. Kaiser S, Lyne J, Agartz I et al.: Individual negative symptoms and domains - relevance for assessment, pathomechanisms and treatment. Schizophrenia Research 2016; 186:39-45

14. Kernberg OF: Neurobiological correlates of object relations theory: The relationship between neurobiological and psychodynamic development. International Forum of Psychoanalysis 2015; 24:38-46

15. Klein M: Some theoretical conclusions regarding the emotional life of the infant. Envy and gratitude and other works 1946-1963. Hogarth Press and the Institute of Psycho-Analysis, New York, 1952

16. Kohut H: The analysis of the self. International Universities Press, Madison, CT, 1971

17. Kohut H: The restoration of the self. International Universities Press, New York, 1977

18. LeDoux JE, Brown R: A higher-order theory of emotional consciousness. PNAS 2017; 114:E2016-E2025

19. Lieb K, Völlm B, Gerta Rücker G et al.: Pharmacotherapy for borderline personality disorder: Cochrane systematic review of randomized trials. The British Journal of Psychiatry 2010; 196:4-12

20. Livesley WJ, Jang KL, Vernon PA: The phenotypic and genetic structure of traits delineating personality disorder. Archives of General Psychiatry 1998; 55:941-948

21. Lyoo IK, Kwon JS, Lee SJ, et al.: Decrease in genu of the corpus callosum in medication- naive, early- onset dysthymia and depressive personality disorder. Biological Psychiatry 2002; 52:1134-1143

22. McAdams DP: Self and identity. In Biswas- Diener R, Diener E (eds.), Noba textbook series: Psychology. Champaign, IL: DEF Publishers, 2017

23. McGlashan T, Grilo C, Sanislow C, et al.: Two- year prevalence and stability of individual DSM- IV criteria for schizotypal, borderline, avoidant, and obsessive-compulsive personality disorders: Toward a hybrid model of 
axis II disorders. American Journal of Psychiatry 2005; 162:883-889

24. Miczek KA, Fish EW: Dopamine, glutamate and aggression. In Schmidt WJ, Reith MEA (eds.), Dopamine and glutamate in psychiatric disorders, 237-263. Humana Press, Totowa, NJ, 2005

25. Newman SW: The medial extended amygdala in male reproductive behavior: $A$ node in the mammalian social behavior network. Annals of the New York Academy of Sciences 1999; 877:242-257

26. Pasquali L, Busceti CL, Fulceri F, et al.: Intracellular pathways underlying the effects of lithium. Behavior Pharmacology 2010; 21:473-492

27. Pazzagli A, Monti MR: Dysphoria and aloneness in borderline personality disorder. Psychopathology 2000; 33:220-226

28. PDM Task Force: Psychodynamic diagnostic manual. Alliance of Psychoanalysis Organizations, Silver Spring, MD, 2006

29. Posternak MA, Zimmerman M: Dual reuptake inhibitors incur lower rates of tachyphylaxis than selective serotonin reuptake inhibitors: A retrospective study. Journal of Clinical Psychiatry 2005; 66:705-707

30. Proust J: What is a mental function? In Brenner A, Gayon $J$ (eds.), Philosophy of science contemporary research in France. Springer, New York, 2009
31. Siegel A, Victoroff J: Understanding human aggression: New insights from neuroscience. International Journal of Law and Psychiatry 2009; 32:209-215

32. Svrakic DM, Whitehead CA, Przybeck TR, et al.: Differential diagnosis of personality disorders by the seven- factor Temperament and Character Inventory. Archives of General Psychiatry 1993; 50:991- 999

33. Svrakic D, Divac-Jovanovic M: The Fragmented Personality: An Integrative, Dynamic, and Personalized Approach to Personality Disorder. Oxford University Press, USA, 2018

34. Svrakic NM, Svrakic DM, Cloninger CR: A general quantitative theory of personality development: Fundamentals of a self- organizing psychobiological complex. Development and Psychopathology 1996; 8:247- 272

35. Svrakic D, Mofsen A, Chockalingam R, et al.: Mechanismbased Pharmacotherapy of Personality Disorder: An Emerging Concept. Manuscript In Preparation

36. Takahashi A, Lee RX, Iwasato TI, et al.: Glutamate input in the dorsal raphe nucleus as a determinant of escalated aggression in male mice. Journal of Neuroscience 2015; 35:6452-6463

37. Victoroff J: The neurpopsychiatry of human aggression. In Sadock BJ, Sadock VA, Ruiz P (eds.), Kaplan and Sadock's comprehensive textbook of psychiatry, 10th ed., 2471-2504. Wolters Kluwer, New York, 2017

Correspondence:

Professor Dragan Svrakic, MD, PhD

Department of Psychiatry, VA medical center St Louis, Washington University School of Medicine

St Louis, MO, USA

E-mail: svrakicd@wustl.edu 